\title{
EMBEDDING RANK ONE SIMPLE GROUPS INTO RANK ONE SIMPLE RIESZ GROUPS
}

\author{
E. PARDO \\ Departamento de Matemáticas, Universidad de Cádiz, Apartado 40, 11510 Puerto Real (Cádiz), Spain \\ e-mail: enrique.pardo@uca.es
}

(Received 14 May, 2001; accepted 5 February, 2002)

A la Laia Crespo Oblanca

\begin{abstract}
We give a method for embedding a large family of partially ordered simple groups of rank one into simple Riesz groups of rank one. In particular, we answer in the affirmative a question of Wehrung, by constructing a torsion-free, simple Riesz group $G$ of rank one containing an interval $D \varsubsetneqq G^{+}$such that $2 D=G^{+}$. We sketch some potential applications of this result in the context of monoids of intervals and K-Theory of rings.
\end{abstract}

2000 Mathematics Subject Classification. Primary 6F20, 20K20; Secondary 16A50, 16D70, 19K14, 46L05.

1. Introduction. In [6, Open Problem 30], Goodearl asks whether every partially ordered simple abelian group can be embedded into a simple Riesz group. Wehrung [18] answers this question in the affirmative. Moreover, he shows that this embedding can be choosen to be cofinal, so that the simple Riesz group arising from his construction is tightly related to the original group. Nevertheless, his argument lies in the scope of Model Theory, and unfortunately it cannot be translated to concrete constructions for given families of groups. In [18, Example 3.14], Wehrung uses his construction to give an example of a torsion-free simple Riesz group $G$ containing an interval $D \neq G^{+}$such that $2 D=G^{+}$, and he asks ([18, Problem 3.15]) whether this example can be realized as a torsion-free Riesz group of rank one (i.e, with positive cone isomorphic to an additive submonoid of $\mathbb{Q}$ ). The existence of such a constructive example of simple Riesz group might have interesting consequences in the context of K-Theory for $C^{*}$-algebras and exchange rings, so that it is interesting to have an answer to Wehrung's Problem.

In this note we answer Wehrung's Problem in the affirmative. For, we start by showing that, for any group $G$ lying within a large family of torsion-free simple partially ordered groups of rank one, it is possible to construct a concrete torsion-free, simple Riesz group of rank one $\widetilde{G}$, as well as a concrete order-embedding from $G$ onto $\widetilde{G}$. Since the original group $G$ appearing in [18 Example 3.14] belongs to this family, we obtain the desired result. Observe that our result is, in fact, a partial answer to [6, Open Problem 30], but a constructive one, and so is different from the complete (but not constructive) answer given by Wehrung. To obtain our result, we

Partially supported by DGESIC grant PB98-0873, by the Comissionat per Universitats i Recerca de la Generalitat de Catalunya, and by PAI III grant FQM-298. 
accurately re-elaborate the construction introduced in [10, Section 3]. Nevertheless, the kind of groups we obtain are quite different from those constructed in [10].

Also, we will explain how these examples apply for producing pathological examples of refinement monoids failing separative cancellation property (see [1]), and how they could be applied for answering some open questions in the context of exchange rings.

Throughout this note we will refer to [6] for notations and definitions on partially ordered abelian groups. We recall here some basic definitions on the context of this note. Given an abelian group $G$, a cone of $G$ is an additive submonoid $P$ of $G$ containing zero, and we say that the cone $P$ is strict if $P \cap(-P)=\{0\}$. A partially ordered abelian group is an abelian group $G$ endowed with a strict cone, called the positive cone of $G$ and denoted by $G^{+}$. A partially ordered abelian group $G$ is said to be directed provided that any element can be written as a difference of two positive elements. Given a partially ordered abelian group $G$, an element $u \in G$ is said to be an order-unit provided that $0 \neq u \in G^{+}$and for each element $x \in G$ there exists $n$ in $\mathbb{N}$ such that $-n u \leq x \leq n u$ (in particular a group with order-unit is directed). A partially ordered abelian group is said to be simple when it is nonzero and every nonzero positive element is an order-unit. A partially ordered abelian group $G$ is said to be (strictly) unperforated provided that for all $x \in G$ and for all $n \in \mathbb{N}=\mathbb{Z}^{+} \backslash\{0\}$, if $n x \in G^{+}\left(0 \neq n x \in G^{+}\right)$, then $x \in G^{+}\left(0 \neq x \in G^{+}\right)$. A partially ordered abelian group is an interpolation group if for all $x_{1}, x_{2}, y_{1}, y_{2} \in G$ such that $\forall i, j x_{i} \leq y_{j}$, there exists an element $z \in G$ such that $\forall i, j x_{i} \leq z \leq y_{j}$. A Riesz group is a directed interpolation group. Following [10], a partially ordered abelian group is $a$ simple component if $G=\left(\mathbb{Z}, G^{+}\right)$and $G$ is directed. Observe that $G$ then is obviously simple. Given $r_{1}, \ldots, r_{l} \in \mathbb{Z}^{+}$, we denote by $\left\langle r_{1}, \ldots, r_{l}\right\rangle$ the additive submonoid of $\mathbb{Z}^{+}$ generated by $\left\{r_{1}, \ldots, r_{l}\right\}$. Given $G, H$ partially ordered abelian groups, a group homomorphism $f: G \rightarrow H$ is a positive morphism if $f\left(G^{+}\right) \subseteq H^{+}$. A positive morph$\operatorname{ism} f: G \rightarrow H$ is an order-embedding if $f$ is a one-to-one morphism, and for every $x \in G, f(x) \in H^{+}$implies $x \in G^{+}$.

2. Building the embedding. We start this section by giving a general expression of the class of groups we will consider. The groups lying in this class can be presented as direct limits in the category of partially ordered abelian groups (see [6, Proposition 1.15]). The basic building blocks of direct systems used in this construction can be built in a recursive way, as follows:

Let $\mathcal{H}=\left\{H_{i}\right\}_{i \geq 1}$ be a sequence of simple components, and let $A=\left\{a_{i}\right\}_{i \geq 1}$ and $B=\left\{b_{j}\right\}_{j \geq 1}$ be sequences of natural numbers, satisfying the following properties, tagged by $(\star)$ :

(i) For every $i \geq 1, a_{i}, b_{i} \geq 2$.

(ii) For every $i \geq 1, a_{i}$ and $b_{i}$ are coprime numbers.

(iii) If we define $b_{0}=1$, then for all $i \geq 1$ we have $b_{i} \in b_{i-1} H_{i}^{+}$.

Given sequences $A, B, \mathcal{H}$ as above, we consider the family

$$
G_{k}(A, B, \mathcal{H})=\left(\mathbb{Z}, G_{k}(A, B, \mathcal{H})^{+}\right)
$$

of partially ordered abelian groups, indexed by $k \geq 1$, whose positive cones are defined recursively as follows:

(i) $G_{1}^{+}(A, B, \mathcal{H})=H_{1}^{+}$.

(ii) For every $k \geq 2, G_{k}^{+}(A, B, \mathcal{H})=a_{k-1} G_{k-1}^{+}(A, B, \mathcal{H})+b_{k-1} H_{k}^{+}$. 
Also, for each $k \geq 1, g_{k, k+1}: G_{k}(A, B, \mathcal{H}) \rightarrow G_{k+1}(A, B, \mathcal{H})$ is the map defined by multiplication by $a_{k}$.

Once we have constructed the direct system

$$
\left\{\left(G_{k}(A, B, \mathcal{H}), G_{k}^{+}(A, B, \mathcal{H}), g_{k, k+1}\right)\right\}_{k \geq 1}
$$

associated with sequences $A, B, \mathcal{H}$, we define the partially ordered abelian group

$$
\left(G(A, B, \mathcal{H}), G^{+}(A, B, \mathcal{H})\right)=\lim _{\rightarrow}\left(G_{k}(A, B, \mathcal{H}), G_{k}^{+}(A, B, \mathcal{H}), g_{k, k+1}\right)
$$

The class that we consider is the one of the groups

$$
\left(G(A, B, \mathcal{H}), G^{+}(A, B, \mathcal{H})\right)
$$

associated with sequences $A, B, \mathcal{H}$ satisfying ( $\star)$.

Definition 2.1. (i) A triple $(A, B, \mathcal{H})$, where $\mathcal{H}=\left\{H_{i}\right\}_{i>1}$ is a sequence of simple components, and $A=\left\{a_{i}\right\}_{i \geq 1}, B=\left\{b_{j}\right\}_{j \geq 1}$ are sequences of natural numbers satisfying ( $\star$ ), is called the data triple $(A, B, \mathcal{H})$.

(ii) Given $(A, B, \mathcal{H})$ a data triple, we say that

$$
\left\{G_{k}(A, B, \mathcal{H})\right\}_{k \geq 1},\left(G(A, B, \mathcal{H}), G^{+}(A, B, \mathcal{H})\right)
$$

is the family of groups associated with the data triple $(A, B, \mathcal{H})$.

REMARK 2.2.

(i) Property ( $\star$ (iii) implies that for every $1 \leq i \leq j, b_{i}$ divides $b_{j}$. So, by property ( $\star$ (ii), for every $1 \leq i \leq j$, g.c.d. $\left(a_{j}, b_{i}\right)=1$.

(ii) Since $A$ and $B$ are nonempty, the above remark implies that there is at least a prime number $p$ coprime with $a_{i}$ for all $i \geq 1$. For, pick $p$ any prime divisor of $b_{1}$. Since for every $1 \leq j$ we have g.c.d. $\left(a_{j}, b_{1}\right)=1$, we conclude that g.c.d. $\left(a_{j}, p\right)=1$ for all $j \geq 1$, as desired.

We proceed to show that the groups $\left(G(A, B, \mathcal{H}), G^{+}(A, B, \mathcal{H})\right)$ are simple groups of rank one. For that, we need to study the basic properties of the family of groups associated with the data triple $(A, B, \mathcal{H})$. First, we introduce some previous definitions and results.

\section{LEMMA 2.3 .}

(1) Let $G_{1}$ and $G_{2}$ be simple components, let $p, q \in \mathbb{N}$ be coprime numbers. Then, $G_{3}=\left(\mathbb{Z}, G_{3}^{+}\right)$with $G_{3}^{+}=p G_{1}^{+}+q G_{2}^{+}$is a simple component.

(2) Let $G_{1}=\left(\mathbb{Z}, G_{1}^{+}\right)$be a partially ordered abelian group, let $M$ be a submonoid of $\mathbb{Z}^{+}$, let $a, b \in \mathbb{N}$ be coprime numbers, and let $G_{2}=\left(\mathbb{Z}, G_{2}^{+}\right)$with $G_{2}^{+}=a G_{1}^{+}+b M$. If $b \in G_{1}^{+}$, then the map $f: G_{1} \rightarrow G_{2}$, defined by multiplication by $a$, is an orderembedding.

Proof. (1) Since $G_{1}$ and $G_{2}$ are simple components, there exist $a \in G_{1}^{+}, b \in G_{2}^{+}$ different prime numbers bigger than both $p$ and $q$. Thus, $p a, q b \in G_{3}^{+}$and g.c.d. $(p a, q b)=1$, whence the result holds (see, for example, [13, Lemma 2.3]). 
(2) Since the underlying group of both $G_{1}$ and $G_{2}$ is $\mathbb{Z}, f$ is an injective group morphism. Moreover, as $a G_{1}^{+} \subseteq G_{2}^{+}, f$ is a positive morphism. Let $x \in G_{1}$ such that $a x \in G_{2}^{+}$. Then, there exist $y \in G_{1}^{+}$and $z \in M$ such that $a x=a y+b z$, i.e., $b z=a(x-y)$. Since g.c.d. $(a, b)=1$, there exists $t \in \mathbb{Z}^{+}$such that $z=a t$. Hence, $a b t=b z=a(x-y)$, whence $x-y=b t$. Since $b \in G_{1}^{+}$, so is $b t=x-y$ for some $t \in \mathbb{Z}^{+}$. Thus, $x \in G_{1}^{+}$, as desired.

The next result is well-known, but we include here a proof for the sake of completeness.

Lemma 2.4. Let $\left\{G_{n}, G_{n}^{+}, f_{n, m}\right\}_{1 \leq n \leq m}$ be a direct system of directed partially ordered abelian groups, let $\left(G, G^{+}\right)$be the direct limit (in the category of partially ordered abelian groups) of this system, and for every $n \geq 1$, let $f_{n}: G_{n} \rightarrow G$ be the canonical map. Then:

(1) If for every $n \geq 1$ the group $\left(G_{n}, G_{n}^{+}\right)$is simple, then so is $\left(G, G^{+}\right)$.

(2) If for every $1 \leq n \leq m$ the map $f_{n, m}: G_{n} \rightarrow G_{m}$ is an order-embedding, then for every $n \geq 1$ the map $f_{n}: G_{n} \rightarrow G$ is an order-embedding.

Proof. Recall that $G^{+}=\bigcup_{i \geq 1} f_{n}\left(G_{n}^{+}\right)$.

(1) Let $x \in G^{+}$be a nonzero positive element, and let $y \in G$ be an arbitrary element. Then, we can assume that there exist $n \in \mathbb{N}, 0 \neq x_{n} \in G_{n}^{+}$and $y_{n} \in G_{n}$ such that $x=f_{n}\left(x_{n}\right)$ and $y=f_{n}\left(y_{n}\right)$. Since $\left(G_{n}, G_{n}^{+}\right)$is simple, there exists $k \in \mathbb{N}$ such that $-k x_{n} \leq y_{n} \leq k x_{n}$. Thus,

$$
-k x=f_{n}\left(-k x_{n}\right) \leq f_{n}\left(y_{n}\right)=y \leq f_{n}\left(k x_{n}\right)=k x,
$$

so we are done.

(2) Fix $n \geq 1$. First, we show that, for every $n \geq 1, f_{n}: G_{n} \rightarrow G$ is an injective map. For, let $x \in \operatorname{ker} f_{n}$. Since $f_{n}(x)=0$, there exists $m \geq n$ such that $f_{n, m}(x)=0=f_{n, m}(0)$. Thus, as $f_{n, m}$ is an injective map, we conclude that $x=0$, as desired.

Now, let $x \in G_{n}$ such that $f_{n}(x) \in G^{+}$. Then there exist $m \geq n$ and $y \in G_{m}^{+}$such that $f_{m}(y)=f_{n}(x)$. Hence, as $f_{m}\left(f_{n, m}(x)\right)=f_{n}(x)=f_{m}(y)$, the injectivity of $f_{m}$ implies that $f_{n, m}(x)=y \in G_{m}^{+}$. Since $f_{n, m}$ is an order embedding, we conclude that $x \in G_{n}^{+}$, which ends the proof.

Let $\mathbb{P}$ be the set of the natural prime numbers. Recall that a generalized integer $\mathfrak{n}$ is a map

$$
\mathfrak{n}: \mathbb{P} \longrightarrow\{0,1,2, \ldots, \infty\}
$$

Usually we write

$$
\mathfrak{n}=\prod_{p \in \mathbb{P}} p^{\mathfrak{n}(p)} .
$$

When $\mathfrak{n}$ is finite (i.e. it never takes the value $\infty$ and it is zero except at finitely many primes), we identify $\mathfrak{n}$ with the integer appearing on the right hand side of (1). For every generalized integer $\mathfrak{n}$, a sequence $\left\{a_{n}\right\}_{n \geq 1}$ of positive integers is associated with $\mathfrak{n}$ when $\mathfrak{n}=\prod_{n \geq 1} a_{n}$. Given a sequence $A=\left\{a_{n}\right\}_{n \geq 1}$, we define $\mathfrak{n}(A)=\prod_{n \geq 1} a_{n}$, so that 
the sequence $A$ is the sequence associated to $\mathfrak{n}(A)$. Notice that the notion of divisibility of integer numbers extends to the context of generalized integers: given $\mathfrak{m}, \mathfrak{n} \in \widetilde{\mathbb{N}}$, we say that $\mathfrak{n} \mid \mathfrak{m}$ if there exists $\mathfrak{n}^{\prime} \in \widetilde{\mathbb{N}}$ such that $\mathfrak{m}=\mathfrak{n} \cdot \mathfrak{n}^{\prime}$, that is, for each $p \in \mathbb{P}$ we have $\mathfrak{m}(p)=\mathfrak{n}(p)+\mathfrak{n}^{\prime}(p)$. Given two generalized integers $\mathfrak{n}, \mathfrak{m}$, we say that $\mathfrak{n}$ and $\mathfrak{m}$ are coprime if for every $p \in \mathbb{P}$ we have $0 \in\{\mathfrak{n}(p), \mathfrak{m}(p)\}$. Let $\mathfrak{n}$ be an infinite generalized integer satisfying, for a fixed $p \in \mathbb{P}$, that $\mathfrak{n}(q)=0$ for every $q \in \mathbb{P} \backslash\{p\}$. Then, we will denote such a generalized integer $\mathfrak{n}$ by $p^{\infty}$. In this case, if $\mathfrak{m}$ is a generalized integer coprime with $p^{\infty}$, we will say that $\mathfrak{m}$ is coprime with $p$ instead of $p^{\infty}$. In the particular case of $2^{\infty}$, we will say that $\mathfrak{m}$ is an odd generalized integer instead of saying that it is coprime with 2 .

Given $\mathfrak{n}$ a generalized integer, we associate to $\mathfrak{n}$ an additive subgroup of $\mathbb{Q}$ containing 1 in the following way: write $\mathbb{Z}_{\mathfrak{n}}=\{a / b:(a \in \mathbb{Z})(b \mid \mathfrak{n})\}$. It is easy to see that $\mathbb{Z}_{\mathfrak{n}}$ is an additive subgroup of $\mathbb{Q}$, by using [10, Lemma 2.3].

Now we are able to state the basic properties of the family of groups associated with a given data triple $(A, B, \mathcal{H})$.

Proposition 2.5. Let $\left\{G_{k}(A, B, \mathcal{H})\right\}_{k \geq 1},\left(G(A, B, \mathcal{H}), G^{+}(A, B, \mathcal{H})\right)$ be the family of groups associated with the data triple $(A, B, \mathcal{H})$. Then:

(1) For every $k \geq 1, G_{k}(A, B, \mathcal{H})$ is a simple component.

(2) For every $k \geq 1$, the map $g_{k, k+1}: G_{k}(A, B, \mathcal{H}) \rightarrow G_{k+1}(A, B, \mathcal{H})$ is an orderembedding.

(3) The group $\left(G(A, B, \mathcal{H}), G^{+}(A, B, \mathcal{H})\right)$ is a simple group of rank one, and for every $k \geq 1$ the canonical map $g_{k}: G_{k}(A, B, \mathcal{H}) \rightarrow G(A, B, \mathcal{H})$ is an order-embedding.

Proof. (1) We proof this by induction on $k$. The case $k=1$ holds because $G_{1}(A, B, \mathcal{H})=H_{1}$. Suppose that the result holds for $k \geq 1$. Then we have

$$
G_{k+1}^{+}(A, B, \mathcal{H})=a_{k} G_{k}^{+}(A, B, \mathcal{H})+b_{k} H_{k+1}^{+} .
$$

$H_{k+1}$ is a simple component by definition, $G_{k}(A, B, \mathcal{H})$ is a simple component by induction hypothesis, and g.c.d. $\left(a_{k}, b_{k}\right)=1$ by property ( $\star$ ) (ii). Thus, $G_{k+1}(A, B, \mathcal{H})$ is a simple component by Lemma 2.3(1). Hence, the induction works, as desired.

(2) If $k=1$, then $G_{1}^{+}(A, B, \mathcal{H})=H_{1}^{+}$, and $b_{1} \in G_{1}^{+}$by property ( $\star$ )(iii). If $k \geq 2$, then we have

$$
G_{k}^{+}(A, B, \mathcal{H})=a_{k-1} G_{k-1}^{+}(A, B, \mathcal{H})+b_{k-1} H_{k}^{+} .
$$

Again by property $(\star)$ (iii) we have $b_{k} \in b_{k-1} H_{k}^{+} \subseteq G_{k}^{+}(A, B, \mathcal{H})$. Hence, the result holds by Lemma $2.3(2)$.

(3) $\left(G(A, B, \mathcal{H}), G^{+}(A, B, \mathcal{H})\right)$ is a simple group by part (1) and Lemma $2.4(1)$. Let $\mathfrak{n}(A)=\prod_{n \geq 1} a_{n}$ be the generalized integer associated with the sequence $A$. Then, according to [10, Lemma 2.4], $G(A, B, \mathcal{H})$ is isomorphic (as an abelian group) to $\mathbb{Z}_{\mathfrak{n}(A)}$. Hence, we conclude that the group $\left(G(A, B, \mathcal{H}), G^{+}(A, B, \mathcal{H})\right)$ is a partially ordered abelian group of rank one. The final assert holds by part (2) and Lemma 2.4(2).

\section{REMARK 2.6.}

(1) Let $(A, B, \mathcal{H})$ be a data triple. By Proposition $2.5(3), G(A, B, \mathcal{H}) \cong \mathbb{Z}_{\mathfrak{n}(A)}$. Under this point of view, $A$ can be considered a "set of denominators" of $G(A, B, \mathcal{H})$. 
(2) Given any prime number $p$ and any infinite generalized integer $\mathfrak{n}$ coprime with $p$, we can construct a data triple $(A, B, \mathcal{H})$ such that the group $G(A, B, \mathcal{H})$ associated with this data triple is isomorphic to $\mathbb{Z}_{\mathfrak{n}}$ (as an abelian group). For, let $A=\left\{a_{n}\right\}_{n \geq 1}$ a sequence associated to $\mathfrak{n}$, let $B=\left\{p^{n}\right\}_{n \geq 1}$, and for a fixed simple component $H$, let $\mathcal{H}=\left\{H_{n}=H\right\}_{n \geq 1}$. Thus, $(A, B, \mathcal{H})$ is a data triple, and by Proposition $2.5(3)$, the group $G(A, B, \mathcal{H})$ associated with this data triple is isomorphic to $\mathbb{Z}_{\mathfrak{n}}$ (as an abelian group). Thus, the class of groups we are considering is quite large.

Now, given the simple group of rank one $G(A, B, \mathcal{H})$, associated with the data triple $(A, B, \mathcal{H})$, we will construct a simple Riesz group of rank one $\widetilde{G}(A, B, \mathcal{H})$ such that $G(A, B, \mathcal{H})$ embeds (as an ordered group) into $\widetilde{G}(A, B, \mathcal{H})$. The idea is to use the results of [10, Section 3] in order to get, for every $k \geq 1$, a simple component $\widetilde{G}_{k}(A, B, \mathcal{H})$ and an order-embedding $\tau_{k}: G_{k}(A, B, \mathcal{H}) \rightarrow \widetilde{G}_{k}(A, B, \mathcal{H})$. Then, we will refine this construction in order to get, for every $k \geq 1$, an order-embedding $h_{k, k+1}$ : $\widetilde{G}_{k}(A, B, \mathcal{H}) \rightarrow \widetilde{G}_{k+1}(A, B, \mathcal{H})$ so that the "obstruction" to get Riesz property on $\widetilde{G}_{k}(A, B, \mathcal{H})$ "can be solved" in $\widetilde{G}_{k+1}(A, B, \mathcal{H})$, and also the diagram ( $(\mathbf{)})$ defined by these groups and maps

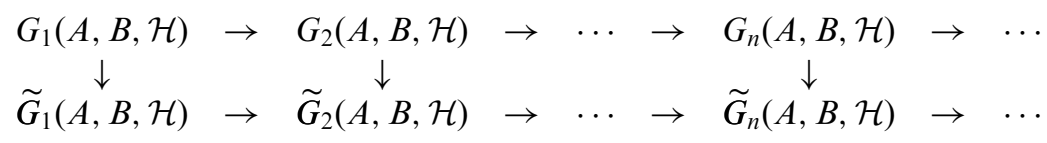

commutes. Thus, if we define

$$
\left(\widetilde{G}(A, B, \mathcal{H}), \widetilde{G}^{+}(A, B, \mathcal{H})\right)=\lim _{\rightarrow}\left(\widetilde{G}_{k}(A, B, \mathcal{H}), \widetilde{G}_{k}^{+}(A, B, \mathcal{H}), h_{k, k+1}\right),
$$

it is easy to see that this group is simple, Riesz, and its rank is one. Moreover, the map $\tau: G(A, B, \mathcal{H}) \rightarrow \widetilde{G}(A, B, \mathcal{H})$ induced by the above diagram turns out to be an order-embedding by Lemma $2.4(2)$.

We proceed to develop this idea. For that, we need some previous definitions and results of [10, Section 3]. We quote them here for convenience of the reader. Recall that, if $G$ is a partially ordered abelian group, we say that an interpolation problem of $G$ is a quadruple of the form $\alpha=\left(x_{1}, x_{2}, y_{1}, y_{2}\right)$ of elements of $G$ satisfying $\left\{x_{1}, x_{2}\right\} \leq_{G}\left\{y_{1}, y_{2}\right\}$, while a solution of $\alpha$ is an element $z \in G$ satisfying $\left\{x_{1}, x_{2}\right\} \leq_{G} z \leq_{G}\left\{y_{1}, y_{2}\right\}$. If $G$ is a simple component, we say that a reduced interpolation problem is an interpolation problem of the form $\left(0, x, y_{1}, y_{2}\right)$ with $0<_{\mathbb{Z}} x<_{\mathbb{Z}} y_{1}<_{\mathbb{Z}} y_{2}$.

Lemma 2.7. ([10, Lemma 3.7]) Let $G=\left(\mathbb{Z}, G^{+}\right)$be a simple component. Then:

(1) For each interpolation problem $\alpha$ of $G$ there exists a reduced interpolation problem $\alpha^{\prime}$ of $G$ and there exists an element $n \in G$ such that if $r \in G$ is a solution of $\alpha^{\prime}$ then $r+n \in G$ is a solution of $\alpha$.

(2) There are only finitely many reduced interpolation problems of $G$ without solution in $G$.

Recall that for every simple component $G$ and every finite list of interpolation problems $\alpha_{1}, \cdots, \alpha_{t}$ of $G, E\left(G ; \alpha_{1}, \cdots, \alpha_{t}\right)$ denotes the set of all positive integers $q$ such that there exists a simple component $H$ satisfying the two following conditions: 
(i) The multiplication map $q$. defines an embedding of ordered groups from $G$ into $H$.

(ii) For all $i \in\{1, \ldots, t\}, q \alpha_{i}$ admits a solution in $H$.

If $\alpha_{1}, \cdots, \alpha_{t}$ is the list of all reduced interpolation problems of $G$ without a solution in $G$ (which is finite because of Lemma 2.7), we write $E(G)$ instead of $E\left(G ; \alpha_{1}, \cdots, \alpha_{t}\right)$.

Lemma 2.8. ([10, Lemma 3.9]) Let $G=\left(\mathbb{Z}, G^{+}\right)$be a simple component, let $\mathfrak{n}$ be an infinite generalized integer, let $\left\{a_{n}\right\}_{n \geq 1}$ be a sequence associated with $\mathfrak{n}$. Then there are arbitrary large $k \in \mathbb{N}$ such that $q_{k}=a_{1} \cdots a_{k}$ belongs to $E(G)$.

The next result is similar to Lemma 2.4.

Lemma 2.9. Let $\left\{G_{n}, G_{n}^{+}, f_{n, m}\right\}_{1 \leq n \leq m}$ and $\left\{H_{n}, H_{n}^{+}, h_{n, m}\right\}_{1 \leq n \leq m}$ be direct systems of directed partially ordered abelian groups, let $\left(G, G^{+}\right)$and $\left(H, H^{+}\right)$be respectively the direct limits (in the category of partially ordered abelian groups) of these systems, and for every $n \geq 1$, let $f_{n}: G_{n} \rightarrow G$ and $h_{n}: H_{n} \rightarrow H$ be the canonical maps. For each $n \geq 1$, let $\tau_{n}: G_{n} \rightarrow H_{n}$ be a map such that $\tau_{m} f_{n, m}=h_{n, m} \tau_{n}$ for all $m \geq n \geq 1$, and let $\tau: G \rightarrow H$ be the induced map. If for every $1 \leq n \leq m$ the maps $f_{n, m}, h_{n, m}$ and $\tau_{n}$ are order-embeddings, then so is $\tau$.

Proof. Notice that, by Lemma 2.4(2), for every $n \geq 1$ the maps $f_{n}$ and $h_{n}$ are order-embeddings.

First, we will prove that $\tau$ is an injective map. For, let $x \in \operatorname{ker} \tau$. Then there exist $n \geq 1$ and $x_{n} \in G_{n}$ such that $f_{n}\left(x_{n}\right)=x$. Thus, $0=\tau(x)=\tau\left(f_{n}\left(x_{n}\right)\right)=\left(h_{n} \circ \tau_{n}\right)\left(x_{n}\right)$. Since $\tau_{n}$ and $h_{n}$ are order-embeddings, so is $h_{n} \circ \tau_{n}$, whence $x_{n}=0$, and hence $x=0$.

Let $x \in G$ such that $\tau(x) \in H^{+}$. Then, there exist $n \geq 1$ and $x_{n} \in G_{n}$ such that $f_{n}\left(x_{n}\right)=x$. Hence, $\tau(x)=\tau\left(f_{n}(x)\right)=\left(h_{n} \circ \tau_{n}\right)\left(x_{n}\right)$. Since $h_{n}$ and $\tau_{n}$ are order-embeddings, we have that $x_{n} \in G_{n}^{+}$. Thus, $x \in G^{+}$, so we are done.

We need the following result, in order to construct the diagram ( $\mathbb{( 1 )}$ in a recursive way.

Proposition 2.10. Let $G_{1}=\left(\mathbb{Z}, G_{1}^{+}\right), G_{2}=\left(\mathbb{Z}, G_{2}^{+}\right), G_{3}=\left(\mathbb{Z}, G_{3}^{+}\right)$be partially ordered abelian groups, and let $a, b \in \mathbb{N}$ be coprime numbers such that $a \cdot: G_{1} \rightarrow G_{2}$ and $b \cdot: G_{1} \rightarrow G_{3}$ are positive morphisms. Let $G_{4}=\left(\mathbb{Z}, G_{4}^{+}\right)$with $G_{4}^{+}=a G_{3}^{+}+b G_{2}^{+}$, and consider the commutative diagram

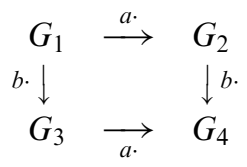

Then:

(1) If $a \cdot: G_{1} \rightarrow G_{2}$ is an order-embedding, then so is $a \cdot: G_{3} \rightarrow G_{4}$.

(2) If $b \cdot: G_{1} \rightarrow G_{3}$ is an order-embedding, then so is $b \cdot: G_{2} \rightarrow G_{4}$

Proof. The argument is similar to that of Lemma 2.3(2).

(1) Clearly, $a \cdot: G_{3} \rightarrow G_{4}$ is a one-to-one morphism. By definition of $G_{4}$, it is also a positive morphism. Let $x \in G_{3}$ such that $a x \in G_{4}^{+}$. Then there exist $y \in G_{3}^{+}$and 
$z \in G_{2}^{+}$such that $a x=a y+b z$, i.e., $a(x-y)=b z$. Since g.c.d. $(a, b)=1$, there exists $t \in \mathbb{Z}^{+}$such that $b z=b a t$, and thus $z=a t$. Since $z \in G_{2}^{+}$and $a \cdot: G_{1} \rightarrow G_{2}$ is an order-embedding, we have that $t \in G_{1}^{+}$, whence $x-y=b t \in G_{3}^{+}$. Thus, $x \in G_{3}^{+}$, as desired.

(2) It is analog to part (1).

Now, we are ready to prove the main result in this note.

Theorem 2.11. Let $\left(G(A, B, \mathcal{H}), G^{+}(A, B, \mathcal{H})\right)$ be the simple rank one group associated with the data triple $(A, B, \mathcal{H})$, and let $\mathrm{n}(A)$ be the generalized integer associated with the sequence $A$. Given any infinite generalized integer $\mathfrak{m}$ coprime with $\mathfrak{n}(A)$, there exist a simple Riesz group of rank one $\left(\widetilde{G}(A, B, \mathcal{H}, \mathfrak{m}), \widetilde{G}^{+}(A, B, \mathcal{H}, \mathfrak{m})\right)$ and a positive morphism

$$
\tau: G(A, B, \mathcal{H}) \rightarrow \widetilde{G}(A, B, \mathcal{H}, \mathfrak{m})
$$

such that:

(1) The group $\widetilde{G}(A, B, \mathcal{H}, \mathfrak{m})$ is isomorphic to $\mathbb{Z}_{\mathfrak{n}(A) \cdot \mathfrak{m}}$ (as abelian groups).

(2) The map $\tau$ is an order-embedding.

Proof. The proof of this Theorem needs a preliminary step, in order to get the necessary groups and morphisms to construct $G(A, B, \mathcal{H}, \mathfrak{m})$ and $\tau$. First notice that, by Remark 2.2(ii), there exist at least an infinite generalized integer $\mathfrak{m}$ coprime with $\mathfrak{n}(A)$. We just need to consider $\mathfrak{m}=p^{\infty}$, where $p$ is a prime number coprime with every $a_{i} \in A$.

Fix $\mathfrak{m}=\prod_{i \geq 1} c_{i}$ an infinite generalized integer coprime with $\mathfrak{n}(A)$. We will show by induction that there exists a commutative diagram $(\dagger)$ of partially ordered abelian groups and positive morphisms

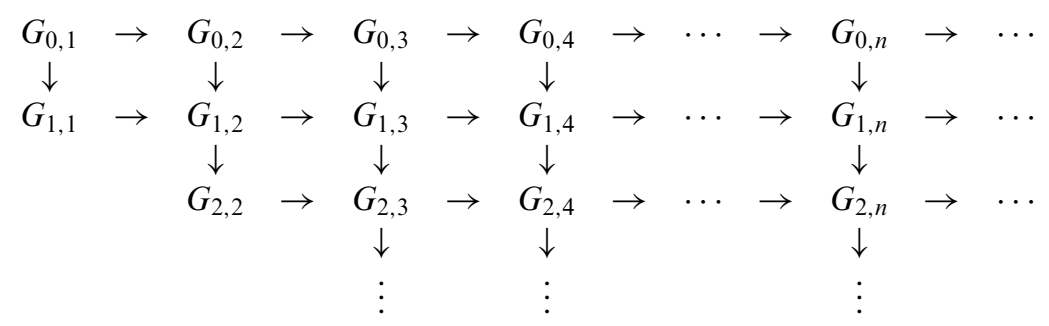

such that:

(i) For every $n \geq 1, G_{0, n}$ is $G_{n}(A, B, \mathcal{H})$, the $n$-th simple component associated with the data triple $(A, B, \mathcal{H})$, and $g_{n, n+1}^{(0)}: G_{0, n} \rightarrow G_{0, n+1}$ is the map $g_{n, n+1}$ : $G_{n}(A, B, \mathcal{H}) \rightarrow G_{n+1}(A, B, \mathcal{H})$ that appears in the construction of $G(A, B, \mathcal{H})$.

(ii) For every $i \geq 0$ and every $j \geq 1$, the group $G_{i, j}=\left(\mathbb{Z}, G_{i, j}^{+}\right)$is a simple component.

(iii) Every map appearing in diagram ( $\dagger$ ) is an order-embedding.

(iv) For every $i \geq 0$, the map $f_{i, i+1}^{(i+1)}: G_{i, i+1} \rightarrow G_{i+1, i+1}$ is given by multiplication by an integer $q_{i}$ satisfying: (a) for every $n \geq 1, \prod_{i=1}^{n} q_{i}$ divides $\mathfrak{m}$; (b) the image of every reduced interpolation problem of $G_{i, i+1}$ admits a solution in $G_{i+1, i+1}$, that is, $q_{i} \in E\left(G_{i, i+1}\right)$. 
Notice that, by Proposition 2.5(1-2), for every $k \geq 1$, the group $G_{0, k}$ is a simple component, and the map $g_{k, k+1}^{(0)}$ is an order-embedding. Now, for $0 \leq i \leq k$, we will construct the groups $G_{i, k}$ and the maps $g_{k, k+1}^{(i)}: G_{i, k} \rightarrow G_{i, k+1}$ and $f_{i, i+1}^{(\bar{k})}: G_{i, k} \rightarrow$ $G_{i+1, k}$, by induction on the second entry of the subindex pair $(i, k)$.

Case $k=1$ : By Lemma 2.8, there exist $l_{1} \in \mathbb{N}$ and a simple component, that we denote $G_{1,1}$, such that the map given by multiplication by $q_{1}=c_{1} \cdots c_{l_{1}}$ defines an order-embedding $f_{0,1}^{(1)}: G_{0,1} \rightarrow G_{1,1}$ such that $q_{1} \in E\left(G_{0,1}\right)$. Thus, $G_{1,1}$ satisfies property (ii), and the map $f_{0,1}^{(1)}$ satisfies properties (iii-iv).

Case $k=2$ : Notice that, by Proposition 2.5(2), the map $g_{1,2}^{(0)}: G_{0,1} \rightarrow G_{0,2}$, given by multiplication by $a_{1} \in A$, is an order-embedding. Since $\mathfrak{n}(A)$ and $\mathfrak{m}$ are coprime, we have that g.c.d. $\left(a_{1}, q_{1}\right)=1$. We define $G_{1,2}=\left(\mathbb{Z}, G_{1,2}^{+}\right)$, with $G_{1,2}^{+}=a_{1} G_{1,1}^{+}+$ $q_{1} G_{0,2}^{+}$. By Lemma 2.3(1), $G_{1,2}$ is a simple component. Now, we define maps $g_{1,2}^{(1)}: G_{1,1} \rightarrow G_{1,2}$ by multiplication by $a_{1}$, and $f_{0,1}^{(2)}: G_{0,2} \rightarrow G_{1,2}$ by multiplication by $q_{1}$. By definition of $G_{1,2}, g_{1,2}^{(1)}$ and $f_{0,1}^{(2)}$, both maps are positive morphisms such that the diagram

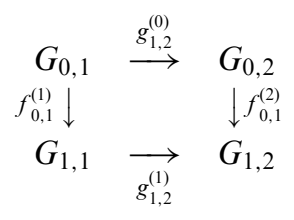

is commutative. Since $g_{1,2}^{(0)}$ and $f_{0,1}^{(1)}$ are order-embeddings by hypothesis, so are $g_{1,2}^{(1)}$ and $f_{0,1}^{(2)}$ because of Proposition 2.10. Now, fix $G_{1,2}$, and set $\mathfrak{m}_{1}=\mathfrak{m} / q_{1}$, that is an infinite generalized integer coprime with $\mathfrak{n}(A)$. Then, by Lemma 2.8 , there exist $l_{2} \in \mathbb{Z}$ and a simple component, that we denote $G_{2,2}$, such that multiplication by $q_{2}=c_{l_{1}+1} \cdots c_{l_{1}+l_{2}}$ defines an order-embedding $f_{1,2}^{(2)}: G_{1,2} \rightarrow G_{2,2}$ such that $q_{2} \in$ $E\left(G_{1,2}\right)$, whence it satisfies properties (iii-iv).

Case $k>2$ : We assume that, for $k \geq 2$ and $i \leq j \leq k$, we have constructed:

- Simple components $G_{i, j}$.

- Order-embeddings $g_{j-1, j}^{(i)}: G_{i, j-1} \rightarrow G_{i, j}$ given by multiplication by $a_{j-1}$.

- Order-embeddings $f_{i-1, i}^{(j)}: G_{i-1, j} \rightarrow G_{i, j}$ given by multiplication by $q_{i}=$ $c_{l_{1}+l_{2}+\cdots+l_{i-1}+1} \cdots c_{l_{1}+l_{2}+\cdots+l_{i}}$, for suitable $l_{1}, \ldots, l_{k}$, in such a way that $f_{i, i+1}^{(i+1)}$ satisfies property (iv).

Moreover, the square diagrams given by these groups and maps,

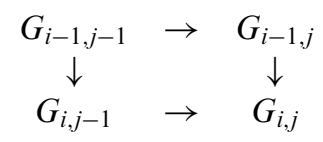

commute.

By Proposition 2.5(2), the map $g_{k, k+1}^{(0)}: G_{0, k} \rightarrow G_{0, k+1}$, given by multiplication by $a_{k}$, is an order-embedding. Since $\mathfrak{n}(A)$ and $\mathfrak{m}$ are coprime, we have g.c.d. $\left(a_{k}, q_{1}\right)=1$. We define $G_{1, k+1}=\left(\mathbb{Z}, G_{1, k+1}^{+}\right)$, with $G_{1, k+1}^{+}=a_{k} G_{1, k}^{+}+q_{1} G_{0, k+1}^{+}$. By Lemma 2.3(1), $G_{1, k+1}$ is a simple component. We define maps $g_{k, k+1}^{(1)}$ : $G_{1, k} \rightarrow G_{1, k+1}$ by multiplication by $a_{k}$, and $f_{0,1}^{(k+1)}: G_{0, k+1} \rightarrow G_{1, k+1}$ by multiplication by $q_{1}$. By definition of $G_{1, k+1}, g_{k, k+1}^{(1)}$ and $f_{0,1}^{(k+1)}$, both maps are positive morphisms such that the diagram 


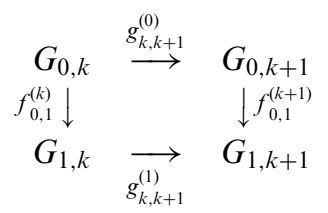

is commutative. Since $g_{k, k+1}^{(0)}$ and $f_{0,1}^{(k)}$ are order-embeddings by hypothesis, so are $g_{k, k+1}^{(1)}$ and $f_{0,1}^{(k+1)}$ because of Proposition 2.10.

Now, we apply the same argument to the simple components $G_{1, k}, G_{1, k+1}$ and $G_{2, k}$, to the order-embedding $g_{k, k+1}^{(1)}: G_{1, k} \rightarrow G_{1, k+1}$ given by multiplication by $a_{k}$, and to the order-embedding $f_{1,2}^{(k)}: G_{1, k} \rightarrow G_{2, k}$ given by multiplication by $q_{2}$. As above, we construct a simple component $G_{2, k+1}$, order-embeddings $g_{k, k+1}^{(2)}: G_{2, k} \rightarrow$ $G_{2, k+1}$ given by multiplication by $a_{k}$, and $f_{1,2}^{(k+1)}: G_{1, k+1} \rightarrow G_{2, k+1}$ given by multiplication by $q_{2}$, so that the diagram

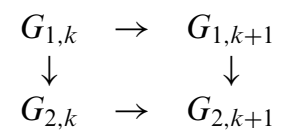

commutes. Repeating this argument for $1 \leq i \leq k$, we construct by recurrence a simple component $G_{i, k+1}$ and a couple of order-embeddings $g_{k, k+1}^{(i)}: G_{i, k} \rightarrow G_{i, k+1}$ given by multiplication by $a_{k}, f_{i-1, i}^{(k+1)}: G_{i-1, k+1} \rightarrow G_{i, k+1}$ given by multiplication by $q_{i}$, so that the diagram

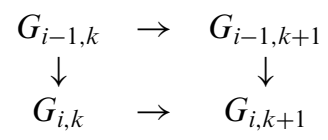

commutes. To end the induction step, we fix $G_{k, k+1}$, and the infinite generalized integer $\mathfrak{m}_{k}=\mathfrak{m} /\left(q_{1} \cdots q_{k}\right)$. Again by Lemma 2.8 , there exist $l_{k+1} \in \mathbb{N}$ and a simple component, that we denote $G_{k+1, k+1}$, such that multiplication by $q_{k+1}=c_{l_{1}+l_{2}+\cdots+l_{k}+1}$ $\cdots c_{l_{1}+l_{2}+\cdots+l_{k+1}}$ defines an order-embedding $f_{k, k+1}^{(k+1)}: G_{k, k+1} \rightarrow G_{k+1, k+1}$ such that $q_{k+1} \in E\left(G_{k, k+1}\right)$, whence it satisfies property (iv). Hence the induction works, so we are done.

Once we stated the existence of diagram $(\dagger)$, we proceed to construct a group satisfying (1-2) in the statement of the Theorem. Recall that, by property (i), for each $n \geq 1, G_{0, n}=G_{n}(A, B, \mathcal{H})$. For each $n \geq 1,\left(\widetilde{G}_{n}(A, B, \mathcal{H}, \mathfrak{m}), \widetilde{G}_{n}^{+}(A, B, \mathcal{H}, \mathfrak{m})\right)$ is the simple component $\left(G_{n, n}, G_{n, n}^{+}\right)$of diagram $(\dagger)$. Thus, for each $n \geq 1$, the composition $\tau_{n}=f_{n-1, n}^{(n)} \circ f_{n-2, n-1}^{(n)} \circ \cdots \circ f_{1,2}^{(n)} \circ f_{0,1}^{(n)}$ defines an order-embedding $\tau_{n}$ : $G_{n}(A, B, \mathcal{H}) \rightarrow \widetilde{G}_{n}(A, B, \mathcal{H}, \mathfrak{m})$. Analogously, for each $n \geq 1$, the composition $h_{n, n+1}=f_{n, n+1}^{(n+1)} \circ g_{n, n+1}^{(n)} \quad$ defines $\quad$ an $\quad$ order-embedding $\quad h_{n, n+1}: \widetilde{G}_{n}(A, B, \mathcal{H}, \mathfrak{m}) \rightarrow$ $\widetilde{G}_{n+1}(A, B, \mathcal{H}, \mathfrak{m})$. Moreover, since the diagram $(\dagger)$ is commutative, so is the diagram ( $($ )

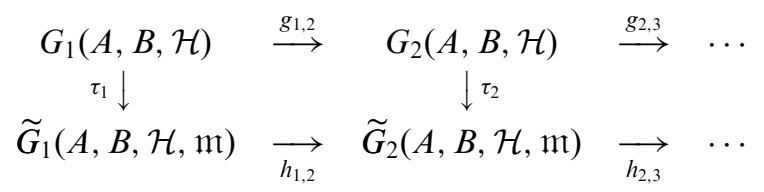


Let

$$
\left(\widetilde{G}(A, B, \mathcal{H}, \mathfrak{m}), \widetilde{G}^{+}(A, B, \mathcal{H}, \mathfrak{m})\right)
$$

be the direct limit (in the category of partially ordered abelian groups) of the direct system

$$
\left\{\widetilde{G}_{n}(A, B, \mathcal{H}, \mathfrak{m}), \widetilde{G}_{n}^{+}(A, B, \mathcal{H}, \mathfrak{m}), h_{n, n+1}\right\}_{n \geq 1},
$$

and let $\tau: G(A, B, \mathcal{H}) \rightarrow \widetilde{G}^{+}(A, B, \mathcal{H}, \mathfrak{m})$ be the map induced by diagram ( $\bullet$ ).

Since for each $n \geq 1$ the map $h_{n, n+1}$ is given by multiplication by $a_{n} \cdot q_{n}$, the sequence associated with the group $\widetilde{G}^{+}(A, B, \mathcal{H}, \mathfrak{m})$ is $\left\{a_{i} q_{i}\right\}_{i \geq 1}$. Notice that $\prod_{i \geq 1} a_{i} q_{i}=\prod_{i \geq 1} a_{i} \prod_{i \geq 1} q_{i}=\mathfrak{n}(A) \mathfrak{m}$. Thus, by [10, Lemma 3.10], $\widetilde{G}(A, B, \mathcal{H}, \mathfrak{m})$ is isomorphic to $\mathbb{Z}_{\mathfrak{n}(A) \mathfrak{m}}$ (as an abelian group). Hence, part (1) holds.

By Lemma 2.4, $\widetilde{G}(A, B, \mathcal{H}, \mathfrak{m})$ is a simple group, and by Lemma 2.9 , $\tau$ is an order-embedding. Since $f_{n, n+1}^{(n+1)}$ and $g_{n, n+1}^{(n)}$ are order-embeddings, property (iv) means that $a_{n} q_{n} \in E\left(\widetilde{G}_{n}(A, B, \mathcal{H}, \mathfrak{m})\right)$. Thus, it is easy to see that $\widetilde{G}(A, B, \mathcal{H}, \mathfrak{m})$ is a $\operatorname{Riesz}$ group, which ends the proof.

As a consequence, we are able to answer in the affirmative [18, Problem 3.15], as a particular case of Theorem 2.11. In the sequel, to simplify the notation, we will denote by $A_{2}$ the constant sequence $\{2\}_{n \geq 1}$, by $B_{9}$ the sequence $\left\{9^{n}\right\}_{n \geq 1}$, and by $\mathcal{H}_{2,7}$ the constant sequence $\{(\mathbb{Z},\langle 2,7\rangle)\}_{n>1}$. Notice that $H=(\mathbb{Z},\langle 2,7\rangle)$ is a simple component by Lemma 2.3(1), and also that $A_{2}$ and $B_{9}$ are sequences of natural numbers satisfying $(\star)$. Hence, $\left(A_{2}, B_{9}, \mathcal{H}_{2,7}\right)$ satisfies Definition 2.1 , so that it is a data triple.

Corollary 2.12. Let $M=\langle 2,7\rangle$, and let $\left(G_{0}, G_{0}^{+}\right)$the simple dense subgroup of $\mathbb{Q}$ generated by the set

$$
G_{0}^{+}=\left\{(k / 2)(9 / 2)^{n} \mid k \in H^{+} \text {and } n \in \mathbb{Z}^{+}\right\} .
$$

Then, for any odd infinite generalized integer $\mathfrak{m}$, there exists a simple Riesz group of rank one $\widetilde{G}_{\mathfrak{m}}$ such that:

(1) $\widetilde{G}_{\mathfrak{m}} \cong \mathbb{Z}_{2^{\infty} \mathfrak{m}}$ as an abelian group.

(2) $G_{0}$ embeds into $\widetilde{G}_{\mathfrak{m}}$ as an ordered group.

Proof. First, notice that the group $\left(G_{0}, G_{0}^{+}\right)$is the one considered in $[\mathbf{1 8}$, Example 3.14]. We construct the group $\left(G_{0}, G_{0}^{+}\right)$as a direct limit, where the groups in the direct system are $\left\{\left(G_{0, n}, G_{0, n}^{+}\right)\right\}_{n \geq 1}$ defined as follows:

1. For every $n \geq 1, G_{0, n}=(1 / 2)^{n} \mathbb{Z}$.

2. $G_{0,1}^{+}=(1 / 2) M$.

3. For every $n \geq 2, G_{0, n}^{+}=\sum_{j=1}^{n}(1 / 2)(9 / 2)^{j-1} M$, or in a more compact way,

$$
G_{0, n}^{+}=G_{0, n-1}^{+}+(1 / 2)(9 / 2)^{n-1} M .
$$

Notice that the natural inclusion maps $h_{n, n+1}: G_{0, n} \longrightarrow G_{0, n+1}$ are order-embeddings.

Now, consider the data triple $\left(A_{2}, B_{9}, \mathcal{H}_{2,7}\right)$, and let

$$
\left\{G_{n}\left(A_{2}, B_{9}, \mathcal{H}_{2,7}\right)\right\}_{n \geq 1}, \quad\left(G\left(A_{2}, B_{9}, \mathcal{H}_{2,7}\right), G^{+}\left(A_{2}, B_{9}, \mathcal{H}_{2,7}\right)\right)
$$

the family of groups associated with this data triple. 
For every $n \geq 1$, the $\operatorname{map} f_{n}: G_{0, n} \rightarrow G_{n}\left(A_{2}, B_{9}, \mathcal{H}_{2,7}\right)$ given by multiplication by $2^{n}$ is an isomorphism of ordered groups. Also, for every $n \geq 1$, if

$$
g_{n, n+1}: G_{n}\left(A_{2}, B_{9}, \mathcal{H}_{2,7}\right) \rightarrow G_{n+1}\left(A_{2}, B_{9}, \mathcal{H}_{2,7}\right)
$$

are the maps appearing in the construction of $G\left(A_{2}, B_{9}, \mathcal{H}_{2,7}\right)$, then $f_{n+1} \circ h_{n, n+1}=$ $g_{n, n+1} \circ f_{n}$, so that the induced map

$$
f: G_{0} \rightarrow G\left(A_{2}, B_{9}, \mathcal{H}_{2,7}\right)
$$

is an isomorphism of ordered groups.

Hence, by Theorem 2.11, for any odd infinite generalized integer $\mathfrak{m}$ there exist a countable, torsion-free, simple Riesz group of rank one

$$
\left(\widetilde{G}\left(A_{2}, B_{9}, \mathcal{H}_{2,7}, \mathfrak{m}\right), \widetilde{G}^{+}\left(A_{2}, B_{9}, \mathcal{H}_{2,7}, \mathfrak{m}\right)\right)
$$

and an order-embedding

$$
\tau: G\left(A_{2}, B_{9}, \mathcal{H}_{2,7}\right) \rightarrow \widetilde{G}\left(A_{2}, B_{9}, \mathcal{H}_{2,7}, \mathfrak{m}\right) .
$$

Set $\widetilde{G}_{\mathfrak{m}}=\widetilde{G}\left(A_{2}, B_{9}, \mathcal{H}_{2,7}, \mathfrak{m}\right)$. Thus, the composition map

$$
g=\tau \circ f: G_{0} \rightarrow \widetilde{G}_{\mathfrak{m}}
$$

give us the desired order-embedding, so we are done.

3. Monoids of intervals. In this section we will use the special properties of the group constructed in Corollary 2.12 to prove some interesting consequences on monoids of intervals. We recall some definitions [11] about monoids of intervals of positive cones of partially ordered abelian groups (specially in the case of simple groups). Since most definitions about abelian monoids are analogous to those of partially ordered abelian groups, we will use them without an explicit definition. For basic definitions and results on abelian monoids, see for example [11], [16] and [17]. Let $G^{+}$be the positive cone of a partially ordered abelian $G$. A nonempty subset $X$ of $G^{+}$is called an interval in $G^{+}$if $X$ is upward directed and order-hereditary. We denote by $\Lambda\left(G^{+}\right)$the set of intervals in $G^{+}$. Note that $\Lambda\left(G^{+}\right)$becomes an abelian monoid with operation defined by $X+Y=\left\{z \in G^{+} \mid z \leq x+y\right.$ for some $x \in X$, $y \in Y$ \}. If $X \in \Lambda\left(G^{+}\right)$, we say that $X$ is countably generated provided that $X$ has a countable cofinal subset (i.e., there is a sequence $\left\{x_{n}\right\}$ of elements in $X$ such that for any $x \in X$ there exists $n \in \mathbb{N}$ such that $\left.x \leq x_{n}\right)$. We denote by $\Lambda_{\sigma}\left(G^{+}\right)$the set of all countably generated intervals in $G^{+}$. Our next result is an immediate consequence of [18, Example 3.14] and Theorem 2.11, but we include a proof for benefit of the reader.

Lemma 3.1. Consider the data triple $\left(A_{2}, B_{9}, \mathcal{H}_{2,7}\right)$, and let

$$
\left(G\left(A_{2}, B_{9}, \mathcal{H}_{2,7}\right), G^{+}\left(A_{2}, B_{9}, \mathcal{H}_{2,7}\right)\right)
$$

be the group associated with this data triple. Let $\mathfrak{m}$ be an odd infinite generalized integer, and let 


$$
\left(\widetilde{G}\left(A_{2}, B_{9}, \mathcal{H}_{2,7}, \mathfrak{m}\right), \widetilde{G}^{+}\left(A_{2}, B_{9}, \mathcal{H}_{2,7}, \mathfrak{m}\right)\right)
$$

be the group constructed in Corollary 2.12. Then there exists a proper countably generated interval $D$ in $\widetilde{G}^{+}\left(A_{2}, B_{9}, \mathcal{H}_{2,7}, \mathfrak{m}\right)$ such that $2 D=\widetilde{G}^{+}\left(A_{2}, B_{9}, \mathcal{H}_{2,7}, \mathfrak{m}\right)$.

Proof. In order to simplify the notation, we will write $\widetilde{G}_{\mathfrak{m}}$ instead of

$$
\widetilde{G}\left(A_{2}, B_{9}, \mathcal{H}_{2,7}, \mathfrak{M}\right) .
$$

Consider the group $\left(G_{0}, G_{0}^{+}\right)$constructed in [18, Example 3.14], and let $g: G_{0} \rightarrow \widetilde{G}_{\mathfrak{m}}$ be the order-embedding constructed in Corollary 2.12. Let $d_{n}=(9 / 2)^{n}$ be elements of $G_{0}^{+}$. According to [18, Example 3.14], for every $n \in \mathbb{Z}^{+}$, the elements $d_{n}, d_{n+1}-d_{n}$ and $2 d_{n+1}-4 d_{n}$ belong to $G_{0}^{+}$. Set $e_{n}:=g\left(d_{n}\right)$. Since $g$ is an orderembedding, we have that $e_{n}, e_{n+1}-e_{n}$ and $2 e_{n+1}-4 e_{n}$ belong to $\widetilde{G}_{\mathfrak{m}}^{+}(*)$. Hence

$$
D=\left\{x \in \widetilde{G}_{\mathrm{m}}^{+} \mid x \leq e_{n} \text { for some } n \in \mathbb{Z}^{+}\right\}
$$

is a countably generated interval. Since there is no $n \in \mathbb{Z}^{+}$such that $2 d_{0} \leq d_{n}$, we have that $2 e_{0} \notin D$, whence $D \neq \widetilde{G}_{\mathfrak{m}}^{+}$. On the other side, since $\widetilde{G}_{\mathfrak{m}}$ is simple, given any $x \in \widetilde{G}_{\mathfrak{m}}^{+}$there exist $m, j \in \mathbb{N}$ such that $x \leq m\left(e_{0}+\cdots+e_{j+1}\right)$. Then, by $(*)$, we have $m\left(e_{0}+\cdots+e_{j+1}\right) \leq(j+1) m e_{j+1}$. Pick $k \in \mathbb{N}$ such that $(j+1) m \leq 2^{k}$. Again by (*) we get $(j+1) m e_{j+1} \leq 2^{k} e_{j+1} \leq 2 e_{j+k}$. Thus, $\widetilde{G}_{\mathfrak{m}}^{+} \subseteq 2 D$, and since $2 D \subseteq \widetilde{G}_{\mathfrak{m}}^{+}$, we are done.

REMARK 3.2. Let $\left(G, G^{+}, 1\right) \subset\left(\mathbb{Q}, \mathbb{Q}^{+}, 1\right)$ be any of the examples of $[\mathbf{1 0}$, Section 3]. By construction, for any $x \in G$, if $x \in \mathbb{Q}^{+}$and $x \geq_{\mathbb{Q}} 1$, then $x \in G^{+}$. As a consequence, if $\left\{x_{n}\right\}$ is any unbounded, strictly ascending chain of $G^{+}$, then the interval generated by $\left\{x_{n}\right\}$, say $X=\left\{x \in G^{+} \mid x \leq x_{n}\right.$ for some $\left.n \in \mathbb{Z}^{+}\right\}$, is equal to $G^{+}$. Hence, $G^{+}$does not contain any interval $D \neq G^{+}$such that $n D=G^{+}$for some positive integer $n$. In this sense, the groups obtained by using Theorem 2.11, and those obtained by using the construction in [10, Section 3], are essentially different.

Recall that an abelian monoid $M$ is separative if it satisfies the weak cancellation condition that for all $a, b$ in $M, a+a=a+b=b+b$ only if $a=b$. Also, an element $x$ of a monoid $M$ is said to be directly finite in $M$, if, for all $0 \neq y \in M, x+y \neq x$; otherwise, $x$ is directly infinite in $M$. If $D$ is a fixed interval in $\Lambda_{\sigma}\left(G^{+}\right)$, we denote by $\Lambda_{\sigma, D}\left(G^{+}\right)$the submonoid of $\Lambda_{\sigma}\left(G^{+}\right)$whose elements are intervals $X \in \Lambda_{\sigma}\left(G^{+}\right)$such that $X \subseteq n D$ for some $n \in \mathbb{N}$, and we denote by $W_{\sigma}^{D}\left(G^{+}\right)$the submonoid of $\Lambda_{\sigma, D}\left(G^{+}\right)$ whose elements are intervals $X \in \Lambda_{\sigma, D}\left(G^{+}\right)$such that there exists $Y \in \Lambda_{\sigma, D}\left(G^{+}\right)$with $X+Y=n D$ for some $n \in \mathbb{N}$. Thus, as an immediate consequence of Lemma 3.1 we have

COROllary 3.3. Let $\mathfrak{m}$ be any odd infinite generalized integer, and let $\widetilde{G}\left(A_{2}, B_{9}, \mathcal{H}_{2,7}, \mathfrak{m}\right)$ and $D$ be respectively the group and the interval constructed in Lemma 3.1. Then,

$$
W_{\sigma}^{D}\left(\widetilde{G}^{+}\left(A_{2}, B_{9}, \mathcal{H}_{2,7}, \mathfrak{m}\right)\right)
$$

is a non-separative refinement monoid. Moreover, 


$$
D \in W_{\sigma}^{D}\left(\widetilde{G}^{+}\left(A_{2}, B_{9}, \mathcal{H}_{2,7}, \mathfrak{m}\right)\right)
$$

is a directly finite element, but $2 D$ is directly infinite, and satisfies $2(2 D)=2 D$.

Proof. In order to simplify the notation, we will write $\widetilde{G}_{\mathfrak{m}}$ instead of

$$
\widetilde{G}\left(A_{2}, B_{9}, \mathcal{H}_{2,7}, \mathfrak{m}\right) .
$$

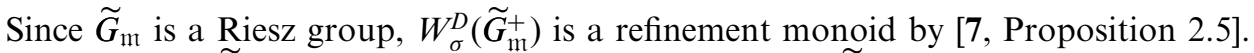
Observe that $\widetilde{G}_{\mathfrak{m}}^{+}$is a countably generated interval in $\widetilde{G}_{\mathfrak{m}}^{+}$(with countably cofinal subset $\{n u \mid n \in \mathbb{N}\}$ for any $0 \neq u \in \widetilde{G}_{m}^{+}$), and also that $\widetilde{G}_{\mathrm{m}}^{+}+0=2 D$. Then, $\widetilde{G}_{\mathfrak{m}}^{+} \in W_{\sigma}^{D}\left(\widetilde{G}_{\mathfrak{m}}^{+}\right)$. By Lemma $3.1, D \widetilde{G}_{\mathfrak{m}}^{+}$and $2 D=\widetilde{G}_{\mathfrak{m}}^{+}$, whence $2 D=D+\widetilde{G}_{\mathfrak{m}}^{+}=2 \widetilde{G}_{\mathfrak{m}}^{+}$, and thus $W_{\sigma}^{D}\left(\widetilde{G}_{\mathfrak{m}}^{+}\right)$is a non-separative monoid.

Suppose that there exists a nonzero interval $X \in W_{\sigma}^{D}\left(\widetilde{G}_{\mathfrak{m}}^{+}\right)$such that $D+X=D$. Then, for every $n \in \mathbb{N}$ we have $D+n X=D$. Let $a \in X$ be a nonzero element. Since $\widetilde{G}_{\mathfrak{m}}$ is a simple group, $a$ is an order-unit. Hence, for any $n \in \mathbb{N}$ there exists $k_{n} \in \mathbb{N}$ such that $d_{n} \leq k_{n} a \in k_{n} X \subset D$. Thus, for all $m, n \in \mathbb{N}$ we have $d_{m}+d_{n} \in$ $k_{m} X+k_{n} X \subset k_{m} X+D=D$, whence $\widetilde{G}_{\mathrm{m}}^{+}=2 D \subseteq D$, contradicting the fact that $D \neq \widetilde{G}_{\mathfrak{m}}^{+}$. As a consequence, $D$ is directly finite. On the other side, $2 D=\widetilde{G}_{\mathfrak{m}}^{+}=$ $2 \widetilde{G}_{\mathfrak{m}}^{+}=2(2 D)$, so we are done.

According to [11, Theorem 3.9], this pathological behavior cannot occur whenever the group $G$ is strictly unperforated. Thus, Remark 3.2 and Corollary 3.3 suggest the following interesting question.

Problem 3.4. Given any countable, torsion-free, simple Riesz group $G$ of rank one that fails to be strictly unperforated, is $W_{\sigma}^{D}\left(G^{+}\right)$always non-separative?

Given a partially ordered abelian group $(G, u)$ with order-unit, we denote by $S_{u}$ the compact convex space of states on $(G, u)$, by $\operatorname{Aff}\left(S_{u}\right)^{+}$the monoid of positive, affine and continuous functions from $S_{u}$ to $\mathbb{R}^{+}$, and by $\phi_{u}: G^{+} \longrightarrow \operatorname{Aff}\left(S_{u}\right)^{+}$the natural evaluation map. Also, let $\operatorname{LAff}_{\sigma}\left(S_{u}\right)^{++}$be the monoid of strictly positive, affine, lower semicontinuous functions from $G^{+}$to $\mathbb{R}^{+}$that are pointwise suprema of increasing sequences of functions in $\operatorname{Aff}\left(S_{u}\right)^{+}$. According to [11, Theorem 3.9], if $G$ is a simple, nonatomic, strictly unperforated Riesz group, $u \in G^{+}$is an order-unit, and $D$ is a nonzero, soft, countably generated interval in $G^{+}$(we say that $X$ is a soft interval in $G^{+}$if for each $x \in X$, there exist $y \in X$ and $n \in \mathbb{N}$ such that $\left.(n+1) x \leq n y\right)$, then the map

$$
\varphi: W_{\sigma}^{D}\left(G^{+}\right) \longrightarrow G^{+} \sqcup W_{\sigma}^{d}\left(S_{u}\right)
$$

given by the rule $\varphi([0, x])=x$ for any $x \in G^{+}$, and by $\varphi(X)=\sup \phi_{u}(X)$ for any $X \in W_{\sigma}^{D}\left(G^{+}\right)$soft interval, is a normalized monoid isomorphism.

Notice that in the case of the group

$$
\left(\widetilde{G}\left(A_{2}, B_{9}, \mathcal{H}_{2,7}, \mathfrak{m}\right), \widetilde{G}^{+}\left(A_{2}, B_{9}, \mathcal{H}_{2,7}, \mathfrak{m}\right)\right)
$$

constructed in Corollary 2.12, since its rank is one, we have that $S_{u}$ is a singleton, so that $\operatorname{Aff}\left(S_{u}\right)^{+}=[0, \infty)$, and $\operatorname{LAff}_{\sigma}\left(S_{u}\right)^{++}=(0, \infty]$. In order to simplify the notation of the following result, we will denote $\widetilde{G}_{\mathfrak{m}}$ instead of $\widetilde{G}\left(A_{2}, B_{9}, \mathcal{H}_{2,7}, \mathfrak{m}\right)$ 
COROLlary 3.5. Let $\mathfrak{m}$ be any odd infinite generalized integer, let $\widetilde{G}_{\mathfrak{m}}$ and $D \subset \widetilde{G}_{\mathfrak{m}}^{+}$be respectively the group and the interval constructed in Lemma 3.1, and let $d=\sup \phi_{u}(D)$. Then the map

$$
\varphi: W_{\sigma}^{D}\left(\widetilde{G}_{\mathfrak{m}}^{+}\right) \longrightarrow \widetilde{G}_{\mathfrak{m}}^{+} \sqcup(0, \infty]
$$

fails to be injective.

Proof. Simply notice that $\varphi(D)=\infty=\varphi\left(\widetilde{G}_{\mathfrak{m}}^{+}\right)$, but $D \neq \widetilde{G}_{\mathfrak{m}}^{+}$.

In a subsequent work [9] it is shown that, under the hypotheses $G$ being a countable, nonatomic, torsion-free, simple Riesz group, the injectivity of $\varphi$ is equivalent to $G$ being strictly unperforated. Moreover, under some mild hypotheses, $\varphi$ turns out to be a monoid epimorphism.

4. Applications to K-Theory. In this section, we observe the potential connections between the above results and some recent works on K-Theory for multiplier rings of both $C^{*}$-algebras of real rank zero [7] and von Neumann regular rings [2], [11]. We briefly recall some definitions on K-Theory. Given a ring $R$, we define $V(R)$ to be the set of isomorphism classes of finitely generated projective right $R$-modules; if $A$ is a finitely generated projective right $R$-module, we denote by $[A]$ the isomorphism class of $A$. We endow $V(R)$ with the structure of an abelian monoid by imposing the operation

$$
[A]+[B]:=[A \oplus B]
$$

for any isomorphism classes $[A]$ and $[B]$. Equivalently [3, Chapter 3], $V(R)$ can be seen as the set of equivalence classes of idempotents in $M_{\infty}(R)$ - the ring of $\omega \times \omega$ matrices over $R$ with only finitely many nonzero entries - with the operation

$$
[e]+[f]:=\left[\left(\begin{array}{ll}
e & 0 \\
0 & f
\end{array}\right)\right]
$$

for idempotents $e, f \in M_{\infty}(R)$. The group $K_{0}(R)$ is the universal group of $V(R)$, and the image of the canonical monoid homomorphism $V(R) \rightarrow K_{0}(R)$ is the positive cone of $K_{0}(R)$, denoted $K_{0}(R)^{+}$. If the Bass' stable rank of $R$ is 1 [1], then $V(R)$ is cancellative, and thus $V(R)=K_{0}(R)^{+}$. Also, if $R$ is a simple ring, then $K_{0}(R)$ is a simple group. In the case of $C^{*}$-algebras with real rank zero, or von Neumann regular rings, or in general exchange rings with stable rank 1, it is well-known that $K_{0}(R)$ is a Riesz group (see [19], [5] and [15], respectively). Thus, when we study simple Riesz groups, we are studying K-Theory of stable rank one rings lying in these classes.

The connection with monoids of intervals was found by Goodearl [7], who applied it to a deep study of the structure of the group $K_{0}$ for the multiplier algebra of a $\sigma$-unital non-unital $C^{*}$-algebra of real rank zero $A$ with stable rank one, such that $K_{0}(A)$ is an unperforated group. Later, Perera [11] used similar techniques for studying the structure of the monoid $V$ for the multiplier algebra of a $\sigma$-unital nonunital, simple, nonartinian $C^{*}$-algebra of real rank zero $A$ with stable rank one, such 
that $V(A)$ is an strictly unperforated monoid. Ara and Perera [2] extended these results to the case of the multiplier ring of a $\sigma$-unital non-unital von Neumann regular ring $R$ with stable rank one, such that $V(R)$ is an strictly unperforated monoid. This case is specially interesting, since by [8], the ring of multipliers of a $\sigma$-unital non-unital von Neumann regular ring is an exchange ring. The fundamental result of both Goodearl [7, Theorem 1.10] and Perera [11, Theorem 2.4] is that, if $R$ is a $\sigma$ unital, non-unital, stable rank one $C^{*}$-algebra of real rank zero (von Neumann regular ring), then there is a monoid isomorphism from $V\left(\mathcal{M}(R),\left[1_{\mathcal{M}(R)}\right]\right)$ to $W_{\sigma}^{D}\left(K_{0}(R)^{+}\right)$for a suitable interval $D$ associated with a fixed approximate unit of $R$, where $\mathcal{M}(R)$ denotes the multiplier ring of $R$ (see [2] for definitions).

Recent results of Villadsen [14], Rørdam and Villadsen [13], and Elliott and Villadsen [4], guarantee the existence of simple $C^{*}$-algebras whose $K_{0}$ groups are simple components. Hence, these results open the possibility of finding a simple $C^{*}$ algebra of real rank zero $A$ with stable rank one such that $\left(K_{0}(A), K_{0}(A)^{+}\right)$is isomorphic to

$$
\left(\widetilde{G}\left(A_{2}, B_{9}, \mathcal{H}_{2,7}, \mathfrak{m}\right), \widetilde{G}^{+}\left(A_{2}, B_{9}, \mathcal{H}_{2,7}, \mathfrak{m}\right)\right)
$$

for some odd infinite generalized integer $\mathfrak{m}$. Suppose that it is possible to construct such a $\sigma$-unital, nonunital, nonartinian, simple, stable rank one $C^{*}$-algebra of real rank zero (von Neumann regular ring) $A$, and suppose that the interval $D$ constructed in Lemma 3.1 is generated by $\left\{\left[e_{n}\right]\right\} \subset V(A)$, where $\left\{e_{n}\right\}$ is a $\sigma$-unit for $A$. Then

Theorem 4.1. Let $A$ be a $\sigma$-unital, nonunital, nonartinian, simple, stable rank one $C^{*}$-algebra of real rank zero (von Neumann regular ring) such that $\left(K_{0}(A), K_{0}(A)^{+}\right)$is isomorphic to

$$
\left(\widetilde{G}\left(A_{2}, B_{9}, \mathcal{H}_{2,7}, \mathfrak{m}\right), \widetilde{G}^{+}\left(A_{2}, B_{9}, \mathcal{H}_{2,7}, \mathfrak{m}\right)\right)
$$

for some odd infinite generalized integer $\mathrm{m}$. Suppose that the interval D constructed in Lemma 3.1 is generated by $\left\{\left[e_{n}\right]\right\} \subset V(A)$, where $\left\{e_{n}\right\}$ is a $\sigma$-unit for $A$. Then, $\mathcal{M}(A)$ is a non separative $C^{*}$-algebra (exchange ring). Moreover, $1_{\mathcal{M}(A)} \in \mathcal{M}(A)$ is a directly finite idempotent, but $2\left[1_{\mathcal{M}(A)}\right]=2\left(2\left[1_{\mathcal{M}(A)}\right]\right) \in V(\mathcal{M}(A))$.

Proof. It is an immediate consequence of [7, Theorem 1.10], [11, Theorem 2.4] and Corollary 3.3 .

Notice that, by Theorem 4.1, if such a regular ring $A$ exists, then $\mathcal{M}(A)$ is a nonseparative exchange ring. Thus we would have a negative answer to a central question of [1]. Also, notice that, by [12, Proposition 3.6], if $A$ is a $\sigma$-unital, nonunital, simple $C^{*}$-algebra, then $\left[1_{\mathcal{M}(A)}\right]=2\left[1_{\mathcal{M}(A)}\right] \in V(\mathcal{M}(A))$ if and only if $R$ is stable (i.e., if $A \cong R \otimes \mathcal{K}$, where $\mathcal{K}$ is the $C^{*}$-algebra of compact operators on a separable Hilbert space). Thus we could have an example of a phenomenon first observed by Rørdam, who provided an explicit example [12, Theorem 3.7, Example 4.3], produced in a different way

COROLlary 4.2. Let $A$ be a $\sigma$-unital, nonunital, nonartinian, simple, stable rank one $C^{*}$-algebra of real rank zero such that $\left(K_{0}(A), K_{0}(A)^{+}\right)$is isomorphic to 


$$
\left(\widetilde{G}\left(A_{2}, B_{9}, \mathcal{H}_{2,7}, \mathfrak{m}\right), \widetilde{G}^{+}\left(A_{2}, B_{9}, \mathcal{H}_{2,7}, \mathfrak{m}\right)\right)
$$

for some odd infinite generalized integer $\mathrm{m}$. Suppose that the interval D constructed in Lemma 3.1 is generated by $\left\{\left[e_{n}\right]\right\} \subset V(A)$, where $\left\{e_{n}\right\}$ is a $\sigma$-unit for $A$. Then, $\mathcal{M}(A)$ is a non-stable algebra, but $M_{2}(\mathcal{M}(A))$ is a stable algebra.

ACKNOWLEDGEMENTS. I thank the referee, whose stimulating report has really improved the original version of this note.

\section{REFERENCES}

1. P. Ara, K. R. Goodearl, K. C. O'Meara and E. Pardo, Separative cancellation for projective modules over exchange rings, Israel J. Math. 105 (1998), 105-137.

2. P. Ara and F. Perera, Multipliers of von Neumann regular rings, Comm. Algebra 28(7) (2000), 3359-3385.

3. B. Blackadar, K-theory for operator algebras, Second Edition, M.S.R.I. Publications 5 (Cambridge Univ. Press, 1998).

4. G. Elliott and J. Villadsen, Perforated ordered $K_{0}$-groups, Canad. J. Math. 52(6) (2000), 1164-1191.

5. K. R. Goodearl, Von Neumann regular rings (Pitman, London, 1979); Second Ed. (Krieger, Malabar, Fl., 1991).

6. K. R. Goodearl, Partially ordered abelian groups with interpolation, Math. Surveys and Monographs 20 (A.M.S., Providence, 1986).

7. K. R. Goodearl, $K_{0}$ of multiplier algebras of $C^{*}$-algebras with real rank zero, $K$ Theory 10 (1996), 419-489. (2000).

8. K. C. O'Meara, The exchange property for row and column-finite matrix, Preprint (2001).

9. F. Ortus and E. Pardo, Monoids of intervals of simple refinement monoids, Preprint

10. E. Pardo, On the representation of simple Riesz groups, Comm. Algebra 26(1) (1998), $155-170$.

11. F. Perera, Ideal structure of multiplier algebras of simple $C^{*}$-algebras with real rank zero, Canad. J. Math. 53(3) (2001), 592-630.

12. M. Rørdam, On sums of finite projections, Operator algebras and operator theory (ed. L. Ge, H. Lin, and S. Zhang), Contemp. Math. 228 (1998), 327-340.

13. M. Rørdam and J. Villadsen, On the ordered $K_{0}$-group of universal free product $C^{*}$-algebras, K-Theory 15 (1998), 307-322.

15. R. B. Warfield, Exchange rings and decompositions of modules, Math. Ann. 199 (1972), 31-36. 43-82.

16. F. Wehrung, Injective positively ordered monoids I, J. Pure Appl. Algebra 83 (1992),

17. F. Wehrung, Restricted injectivity, transfer property and decompositions of separative positively ordered monoids, Comm. Algebra 22(5) (1994), 1747-1781.

18. F. Wehrung, Embedding simple commutative monoids into simple refinement monoids, Semigroup Forum 56 (1998), 104-129.

19. S. Zhang, Diagonalizing projections in multiplier algebras and in matrices over a $C^{*}$ algebra, Pacific J. Math. 145 (1990), 181-200. 